\title{
LOOMIS-SIKORSKI THEOREM FOR MONOTONE $\sigma$-COMPLETE EFFECT ALGEBRAS
}

\author{
ANATOLIJ DVUREČENSKIJ \\ (Received 19 April 2004; revised 2 December 2004) \\ Communicated by B. A. Davey
}

\begin{abstract}
We show that monotone $\sigma$-complete effect algebras under some conditions are $\sigma$-homomorphic images of effect-tribes (as monotone $\sigma$-complete effect algebras), which are nonempty systems of fuzzy sets closed under complements, sums of fuzzy sets less than 1, and containing all pointwise limits of nondecreasing fuzzy sets. Because effect-tribes are generalizations of Boolean $\sigma$-algebras of subsets, we present a generalization of the Loomis-Sikorski theorem for such effect algebras. We show that we can choose an effect-tribe to be a system of affine fuzzy sets. In addition, we present a new version of the Loomis-Sikorski theorem for $\sigma$-complete MV-algebras.
\end{abstract}

2000 Mathematics subject classification: primary 06D35, 03G12, 03B50.

Keywords and phrases: effect algebra, monotone $\sigma$-complete effect algebra, MV-algebra, effect-tribe, tribe, Riesz decomposition property, Loomis-Sikorski theorem, po-group, $\ell$-group, affine function.

\section{Introduction}

Effect algebras were introduced in 1993 by Foulis and Bennett [6] as an additive counterpart to D-posets introduced by Kôpka and Chovanec [8]. These algebras are generalizations of MV-algebras introduced by Chang [1] in 1958 as a generalization of Boolean algebras. MV-algebras arise from many valued logic of Łukasiewicz in the same manner as Boolean algebras arise from classical two-valued logic. Effect algebras were inspired by mathematical foundations of quantum mechanics, for an overview on effect algebras see [4]. One of the most important example of effect algebras studied in quantum mechanics is the system $\mathscr{E}(H)$ of all Hermitian operators

The paper has been supported by the grant VEGA No. 2/3163/23 SAV and by Science and Technology Assistance Agency under the contract No. APVT-51-032002.

(C) 2005 Australian Mathematical Society 1446-7887/05 \$A2.00+0.00 
$A$ on a Hilbert space $H$ such that $O \leq A \leq I$, where $O$ and $I$ are the zero and identity operator, respectively, on $H$.

Effect algebras satisfying the Riesz decomposition property ((RDP) in abbreviation) can be represented as intervals in Abelian interpolation po-groups with strong unit, [12], and MV-algebras as intervals in Abelian $\ell$-group with strong unit, [10].

The Loomis-Sikorski theorem for $\sigma$-complete MV-algebras was proved independently by Mundici [11] and the author [3]. It was shown that they are always $\sigma$ homomorphic images of tribes, which are $\sigma$-complete $\mathrm{MV}$-algebras of fuzzy sets.

In the present paper, we generalize the Loomis-Sikorski theorem for a class of monotone $\sigma$-complete effect algebras proving that they are $\sigma$-homomorphic images of effect-tribes (as monotone $\sigma$-complete effect algebras) which are monotone $\sigma$ complete effect algebras of fuzzy sets. We prove that we can choose effect-tribes to be systems of affine fuzzy sets on convex, compact, Hausdorff topological spaces. We show that the class of our monotone $\sigma$-complete effect algebras is in fact always the class of all $\sigma$-complete $\mathrm{MV}$-algebras. Moreover, using the result, we present a new version of the Loomis-Sikorski theorem for $\sigma$-complete $\mathrm{MV}$-algebras.

\section{Effect algebras and states}

An effect algebra is, by [6], a partial algebra $E=(E ;+, 0,1)$ with a partially defined operation + and two constant elements 0 and 1 such that, for all $a, b, c \in E$,

(i) $a+b$ is defined in $E$ if and only if $b+a$ is defined, and in such the case $a+b=b+a$;

(ii) $a+b,(a+b)+c$ are defined if and only if $b+c$ and $a+(b+c)$ are defined, and in such the case $(a+b)+c=a+(b+c)$;

(iii) for any $a \in E$, there exists a unique element $a^{\prime} \in E$ such that $a+a^{\prime}=1$;

(iv) if $a+1$ is defined in $E$, then $a=0$.

If we define $a \leq b$ if and only if there exists an element $c \in E$ such that $a+c=b$, then ' $\leq$ ' is a partial ordering, and we write $c:=b-a$. It is clear that $a^{\prime}=1-a$ for any $a \in E$.

For example, if $(G, u)$ is an Abelian po-group with a strong unit $u$, and if

$$
\Gamma(G, u):=\{g \in G: 0 \leq g \leq u\}
$$

is endowed with the restriction of the group addition + , then $(\Gamma(G, u) ;+, 0, u)$ is an effect algebra. (An element $u \in G^{+}$is said to be a strong unit for a po-group $G$, if given an element $g \in G$, there is an integer $n \geq 1$ such that $-n u \leq g \leq n u$.)

We say that an effect algebra $E$ satisfies (i) the Riesz interpolation property, (RIP) for short, if, for all $x_{1}, x_{2}, y_{1}, y_{2}$ in $E, x_{i} \leq y_{j}$ for all $i, j$ implies there exists an 
element $z \in E$ such that $x_{i} \leq z \leq y_{j}$ for all $i, j$; (ii) the Riesz decomposition property, (RDP) for short, if $x \leq y_{1}+y_{2}$ implies that there exist two elements $x_{1}, x_{2} \in$ with $x_{1} \leq y_{1}$ and $x_{2} \leq y_{2}$ such that $x=x_{1}+x_{2}$.

We recall that (1) if $E$ is a lattice, then $E$ has trivially (RIP), the converse is not true; (2) $E$ has (RDP) if and only if, [4, Lemma 1.7.5], $x_{1}+x_{2}=y_{1}+y_{2}$ implies there exist four elements $c_{11}, c_{12}, c_{21}, c_{22} \in E$ such that $x_{1}=c_{11}+c_{12}, x_{2}=c_{21}+c_{22}$, $y_{1}=c_{11}+c_{21}$, and $y_{2}=c_{12}+c_{22}$; (3) (RDP) implies (RIP), but the converse is not true (for example, if $E=L(H)$, the system of all closed subspaces of a Hilbert space $H$, then $E$ is a complete lattice but without (RDP)). On the other hand, every finite poset with (RIP) is a lattice.

A partially ordered Abelian group $(G ;+, 0)$ is said to satisfy the Riesz decomposition property provided, given $x, y_{1}, y_{2}$ in $G^{+}$such that $x \leq y_{1}+y_{2}$, there exist $x_{1}, x_{2}$ in $G^{+}$such that $x=x_{1}+x_{2}$ and $x_{j} \leq y_{j}$ for each $j$. This condition is equivalent by [7, Proposition 2.1] with the following two equivalent conditions:

(a) Given $x_{1}, x_{2}, y_{1}, y_{2}$ in $G$ such that $x_{i} \leq y_{j}$ for all $i, j$, there exists $z$ in $G$ such that $x_{i} \leq z \leq y_{j}$ for all $i, j$.

(b) Given $x_{1}, x_{2}, y_{1}, y_{2}$ in $G^{+}$such that $x_{1}+x_{2}=y_{1}+y_{2}$, there exist $z_{11}, z_{12}, z_{21}, z_{22}$ in $G^{+}$such that $x_{i}=z_{i 1}+z_{i 2}$ for each $i$ and $y_{j}=z_{1 j}+z_{2 j}$ for each $j$.

According to [7], such a group $G$ with the Riesz decomposition property is said to be the interpolation group.

Ravindran [12] ([4, Theorem 1.17.17]) proved the following important result.

THEOREM 2.1. Let $E$ be an effect algebra with (RDP). Then there exists a unique (up to isomorphism of Abelian po-groups with strong unit) interpolation group $(G, u)$ with strong unit such that $\Gamma(G, u)$ is isomorphic with $E$.

REMARK 2.2. We recall that in Theorem 2.1 all meets and joins from $E$ are preserved in $(G, u)$, see [5, Proposition 6.3]. Moreover, there is also a categorical equivalence among the category of effect algebras with (RDP) and the category of interpolation Abelian po-groups with strong unit. The functor is $\Gamma:(G, u) \rightarrow \Gamma(G, u)$ from $(2.1)$.

A very important family of effect algebras are MV-algebras which were introduced by Chang [1].

We recall that an MV-algebra is an algebra $M=\left(M ; \oplus, \odot,{ }^{*}, 0,1\right)$ of type $(2,2,1,0,0)$ such that, for all $a, b, c \in M$, we have

(i) $a \oplus b=b \oplus a$;

(ii) $(a \oplus b) \oplus c=a \oplus(b \oplus c)$;

(iii) $a \oplus 0=a$;

(iv) $a \oplus 1=1$;

(v) $\left(a^{*}\right)^{*}=a$; 
(vi) $a \oplus a^{*}=1$;

(vii) $0^{*}=1$;

(viii) $\left(a^{*} \oplus b\right)^{*} \oplus b=\left(a \oplus b^{*}\right)^{*} \oplus a$.

If we define a partial operation + on $M$ in such a way that $a+b$ is defined in $M$ if and only if $a \leq b^{*}$ and then we set $a+b:=a \oplus b$, then $(M ;+, 0,1)$ is an effect algebra with (RDP). According to [10], every MV-algebra is isomorphic with the set $\Gamma(G, u)$, where $(G, u)$ is an $\ell$-group with strong unit and $a \oplus b=(a+b) \wedge u$, $a \odot b=0 \vee(a+b-u)$, and $a^{*}=u-a(a, b \in \Gamma(G, u))$.

It is worthy to recall that an effect algebra $E$ with (RDP) can be converted into an MV-algebra (in which both original and induced + coincide) if and only if $E$ is a lattice, see [4].

A state on an effect algebra $E$ is any mapping $s: E \rightarrow[0,1]$ such that (i) $s(1)=1$, and (ii) $s(a+b)=s(a)+s(b)$ whenever $a+b$ is defined in $E$. A state $s$ is said to be $\sigma$-additive if $s\left(a_{n}\right) \nearrow s(a)$ whenever $a_{n} \nearrow a$ in $E$ (that is, $a_{n} \leq a_{n+1}$ for any $n$ and $\left.\bigvee_{n} a_{n}=a\right)$. We denote by $\mathscr{S}(E)$ the set of all states on $E$. It can happen that $\mathscr{S}(E)$ is empty. In important cases, for example when $E$ satisfies (RDP), $\mathscr{S}(E)$ is nonempty. $\mathscr{S}(E)$ is always a convex set. A state $s$ is said to be extremal if $s=\lambda s_{1}+(1-\lambda) s_{2}$ for $\lambda \in(0,1)$ implies $s=s_{1}=s_{2}$. By $\operatorname{Ext}_{\mathscr{S}}(E)$ we mean the set of all extremal states of $\mathscr{S}(E)$. We say that a net of states, $\left\{s_{\alpha}\right\}$, on $E$ converges weakly to a state $s$ on $E$ if $s_{\alpha}(a) \rightarrow s(a)$ for any $a \in E$. Then $\mathscr{S}(E)$ is a compact Hausdorff topological space and every state on $E$ lies in the weak closure of the convex hull of the extremal states as it follows from the Krein-Mil'man theorem.

Let $(G, u)$ be an Abelian po-group with strong unit. By a state on $(G, u)$ we mean any mapping $s: G \rightarrow \mathbb{R}$ such that (i) $s(g+h)=s(g)+s(h)$ for all $g, h \in G$; (ii) $s\left(G^{+}\right) \subseteq \mathbb{R}^{+}$, and (iii) $s(u)=1$. We denote by $\mathscr{S}(G, u)$ the set of all states and by $\operatorname{Ext}_{\mathscr{S}}(G, u)$ the set of all extremal states, respectively, on $(G, u)$. According to [7, Corollary 4.4], $\mathscr{S}(G, u) \neq \emptyset$ whenever $u>0$. In a similar way as for effect algebras we define the weak convergence of states on $(G, u)$, and analogously, $\mathscr{S}(G, u)$ is a compact Hausdorff topological space and every state on $(G, u)$ is a weak limit of a net of convex combinations from $\operatorname{Ext}_{\mathscr{S}}(G, u)$.

If $E=\Gamma(G, u)$, where $(G, u)$ is an interpolation po-group, then from Remark 2.2, every state on $E$ can be extended to a unique state on $(G, u)$, and conversely, the restriction of any state on $(G, u)$ to $E$ gives a state on $E$. Therefore, if $E$ satisfies (RDP), $\mathscr{S}(E) \neq \emptyset$. In addition, extremal states on $E$ are restrictions of extremal states on $(G, u)$, and the space $\mathscr{S}(E)$ is affine homeomorphic with $\mathscr{S}(G, u)$ and the space $\operatorname{Ext}_{\mathscr{S}}(E)$ is affine homeomorphic with $\operatorname{Ext}_{\mathscr{S}}(G, u)$.

Moreover, if $E$ is an MV-algebra, then $\operatorname{Ext}_{\mathscr{S}}(E)$ is compact. This is not always true for effect algebras with (RDP), see for example [7, Example 6.10]. We recall that if $E$ is an MV-algebra, then a state $s$ on $E$ is extremal if and only if $s(x \wedge y)=\min \{s(x), s(y)\}$, $x, y \in E$, or equivalently, if $s(x+y)=\min \{s(x)+s(y), 1\}, x, y \in E$. 
A poset $E$ satisfies

(i) countable interpolation property provided that for any two sequences $\left\{x_{i}\right\}$ and $\left\{y_{j}\right\}$ of elements of $E$ such that $x_{i} \leq y_{j}$ for all $i, j$, there exists an element $z \in E$ such that $x_{i} \leq z \leq y_{j}$ for all $i, j$.

(ii) is monotone $\sigma$-complete provided that for every ascending (descending) sequence $x_{1} \leq x_{2} \leq \cdots\left(x_{1} \geq x_{2} \geq \cdots\right)$ in $E$ which is bounded above (below) in $E$ has a supremum (infimum) in $E$.

THEOREM 2.3. Let $(G, u)$ be an interpolation group with strong unit.

(1) $(G, u)$ has countable interpolation if and only if $E=\Gamma(G, u)$ has countable interpolation.

(2) $(G, u)$ is monotone $\sigma$-complete if and only if $E=\Gamma(G, u)$ is monotone $\sigma$ complete.

(3) If $E=\Gamma(G, u)$ is monotone $\sigma$-complete, then $E$ has countable interpolation.

An ideal of an effect algebra $E$ is a nonempty subset $l$ of $E$ such that (i) $x \in E$, $y \in I, x \leq y$ imply $x \in I$, and (ii) if $x, y \in I$ and $x+y$ is defined in $E$, then $x+y \in I$. We recall that an $o$-ideal of a po-group $G$ is any directed convex subgroup of $G$. We denote by $\mathscr{I}(G, u)$ the set of all o-ideals of $G$.

Let $I$ be a (proper) ideal of an effect algebra $E$ with (RDP). We define a relation $\sim$, on $E$ via $a \sim, b$ if and only if $a-e=b-f$ for some $e, f \in I$. According to [4, Section 3.1.2], we have that $\sim$, is an equivalence such that

(i) $a+b \in E, a_{1}+b_{1} \in E, a \sim_{I} a_{1}, b \sim_{I} b_{1}$ imply $(a+b) \sim_{I}\left(a_{1}+b_{1}\right)$;

(ii) $a \sim, b$ implies $a^{\prime} \sim_{l} b^{\prime}$;

(iii) $a+b \in E, c \sim_{I} a$ imply there exists an element $d \in E$ such that $d \sim_{I} b$ and $d+c \in E$;

(iv) $a+b, a_{1}+b_{1} \in E, a_{1} \sim_{l} a,\left(a_{1}+b_{1}\right) \sim_{l}(a+b)$ imply $b_{1} \sim_{l} b$.

If we define $a / I:=[a]:=[a]_{I}:=\left\{b \in E: b \sim_{l} a\right\}$, then $E / I:=\left\{[a]_{l}: a \in E\right\}$ is an effect algebra, where $[a]+[b]=[c]$ if and only if there exist $a_{1} \in[a], b_{1} \in[b]$, $c_{1} \in[c]$ such that $a_{1}+b_{1}=c_{1}$. Moreover, $E / I$ satisfies (RDP).

The following result was proved in [2].

THEOREM 2.4. Let $(G, u)$ be a interpolation po-group with strong unit and let $E=\Gamma(G, u)$. For any ideal $I$ of $E$, we assign

$$
\phi_{G}(I)=\left\{x \in G: \exists x_{i}, y_{j} \in I, \quad x=x_{1}+\cdots+x_{n}-y_{1}-\cdots-y_{m}\right\} .
$$

Then $\phi_{G}(I)$ is an o-ideal of $(G, u)$. The mapping $\phi_{G}$ defines a one-to-one mapping preserving the set-theoretical inclusion. The inverse mapping $\psi_{G}$ is given by

$$
\psi_{G}(K):=K \cap[0, u], K \in \mathscr{I}(G, u) .
$$


Moreover, $E / I \cong \Gamma\left(G / \phi_{G}(I), u / \phi_{G}(I)\right)$.

A nonempty set $X$ of $\mathscr{S}(E)$ is said to be (i) determining if $s(a)=s(b)$ for all $s \in X$ implies $a=b$, and (ii) order-determining if $s(a) \leq s(b)$ for all $s \in X$ implies $a \leq b$.

LEMMA 2.5. Let $E$ be an effect algebra with (RDP) satisfying the countable interpolation. Let $X$ be a nonempty compact subset of $\operatorname{Ext}_{\mathscr{S}}(E)$. Let $a, b \in E$. The following two statements are equivalent:

(i) $s(a)=0$ for each $s \in X$ implies $a=0$;

(ii) $s(a)=s(b)$ for each $s \in X$ implies $a=b$.

Proof. Assume that $E=\Gamma(G, u)$. For any $s \in X$, let $\hat{s}$ be the unique extension of $s$ onto $(G, u)$, and let us set $\hat{X}=\{\hat{s}: s \in X\}$. Then $\hat{X}$ is a nonempty compact set of $\operatorname{Ext}_{\mathscr{S}}(G, u)$. We define $\operatorname{Ker}(X)=\{a \in E: s(a)=0$ for all $s \in X\}$, and $\operatorname{Ker}(\hat{X})=\{g \in G: \hat{s}(g)=0$ for all $s \in X\}$. Then $\operatorname{Ker}(X)$ is an ideal of $E$ and by [7, Theorem 16.19], $\operatorname{Ker}(\hat{X})$ is an o-ideal of $(G, u)$. Applying Theorem 2.4, we have $\phi_{G}(\operatorname{Ker}(X))=\operatorname{Ker}(\hat{X})$.

It is clear that (ii) $\Rightarrow$ (i). Assume now (i) and let $s(a)=s(b)$ for any $s \in X$. Then $\hat{s}(a-b)=0$ for every $s \in X$. Since $\operatorname{Ker}(X)=\{0\}$, we have $\operatorname{Ker}(\hat{X})=\{0\}$. This means that $a-b \in \operatorname{Ker}(\hat{X})$.

\section{Affine continuous functions}

Let $K$ be a convex subset of a real vector space. A mapping $f: K \rightarrow \mathbb{R}$ is said to be affine if, for all $x, y \in K$ and any $\lambda \in[0,1]$, we have $f(\lambda x+(1-\lambda) y)=$ $\lambda f(x)+(1-\lambda) f(y)$.

Given a compact convex set $K$ in a topological vector space, we denote by $\operatorname{Aff}(K)$ the collection of all affine continuous functions on $K$. Of course, $\operatorname{Aff}(K)$ is a partially ordered group with strong unit which is a subgroup of $C(K)$, the system of all continuous real-valued functions on $K$. Unlike $C(K)$, the space $\operatorname{Aff}(K)$ need to be a lattice. Indeed, according to [7, Theorem 11.21] it is possible to show that $\operatorname{Aff}(\mathscr{S}(G, u))$ is a lattice if and only if $\operatorname{Ext}_{\mathscr{S}}(G, u)$ is compact.

Evaluation at any point $x \in G$ defines a map $\hat{x}: \mathscr{S}(G, u) \rightarrow \mathbb{R}$ such that the mapping $\hat{x}$ is affine and continuous. Hence, the function $\phi(x):=\hat{x}$ defines a map

$$
\phi: G \rightarrow \operatorname{Aff}(\mathscr{S}(G, u))
$$

called a natural mapping from $G$ to $\operatorname{Aff}(\mathscr{S}(G, u)$ ) (that is, $\hat{x}(s)=s(x)$ for all $s \in \mathscr{S}(G, u))$. As each $\hat{x}$ restricts to a continuous real-valued function on the set 
extremal states, $\operatorname{Ext}_{\mathscr{S}}(G, u)$, we obtain again a mapping $\psi: G \rightarrow C\left(\operatorname{Ext}_{\mathscr{S}}(G, u)\right)$ where $\psi(x)(s)=s(x)$ for each extremal state $s$ on $(G, u)$.

It is interesting to recall that given a compact convex subset $K$ of a locally convex Hausdorff space, the state space $\mathscr{S}:=\mathscr{S}(\operatorname{Aff}(K), 1)$ is affine homeomorphic with $K$ under the evaluation map $\psi: K \rightarrow \mathscr{S}$ (this follows from Theorem 2.1 and [7, Theorem 7.1]).

If $s$ is a state on a po-group $(G, u)$ with strong unit, by [7, Lemma 4.21], $s(G)=$ $\{s(g): g \in G\}$ is a subgroup of the group $\mathbb{R}$ of all real numbers which is either cyclic or dense. In the first case $s$ is said to be discrete. In such a case, $s(G)=(1 / n) \mathbb{Z}$ for some integer $n \geq 1$.

A state $s$ on an effect algebra $E$ is said to be discrete if $s(E)=\{s(a): a \in E\} \subseteq$ $\{0,1 / n, 2 / n, \ldots, n / n\}$ for some integer $n \geq 1$. It can happen that $s(E)$ is a proper subset of $\{0,1 / n, 2 / n, \ldots, n / n\}$. Indeed, let $E=\left\{0, a, a^{\prime}, 1\right\}$, and let $s(a)=0.3$ and $s\left(a^{\prime}\right)=0.7$.

We now show that there is a one-to-one correspondence among the discrete states on $E$ and $(G, u)$, respectively.

PROPOSITION 3.1. Let $E=\Gamma(G, u)$ be an effect algebra with (RDP). Then a state $s$ on $E$ is discrete if and only if its extension $\hat{s}$ on $(G, u)$ is discrete.

PROOF. If $\hat{s}$ is discrete, so is easily $s$. Conversely, let $s$ be discrete. That is $s(E) \subseteq\{0,1 / n, 2 / n, \ldots, n / n\}$ for some integer $n \geq 1$; let $n$ be the smallest one. We suppose that $s(E)=\left\{0, k_{1} / n, \ldots, k_{m} / n, 1\right\}$, where $1 \leq k_{1}<\cdots<k_{m} \leq n$. Since $n$ is minimal, this implies that the greatest common divisor of $n, k_{1}, \ldots, k_{m}$ is 1 . From the elementary arithmetic, this yields that there are integers $a_{0}, a_{1}, \ldots, a_{m} \in \mathbb{Z}$ such that $a_{0} n+a_{1} k_{1}+\cdots+a_{m} k_{m}=1$. Therefore, $1 / n \in \hat{s}(G)$, that is, $\hat{s}(G)=(1 / n) \mathbb{Z}$.

The following representation theorem for monotone $\sigma$-complete interpolation groups with strong unit is from [7, Corollary 16.15].

THEOREM 3.2. Let $(G, u)$ be a nonzero monotone $\sigma$-complete interpolation group with strong unit and set

$$
A=\left\{f \in \operatorname{Aff}(\mathscr{S}(G, u)): f(s) \in s(G) \text { for all discrete } s \in \operatorname{Ext}_{\mathscr{S}}(G, u)\right\}
$$

Then the natural mapping $\phi: G \rightarrow \operatorname{Aff}(\mathscr{S}(G, u))$ provides an isomorphism of $(G, u)$ onto $(A, 1)$ (as ordered groups with strong unit).

Let $E$ and $F$ be two effect algebras. A mapping $h: E \rightarrow F$ is said to be a homomorphism if (i) $h(a+b)=h(a)+h(b)$ whenever $a+b$ is defined in $E$, and (ii) $h(1)=1$. A bijective homomorphism $h$ such that $h^{-1}$ is a homomorphism is said to be an isomorphism of $E$ and $F$. If $E$ and $F$ are monotone $\sigma$-complete effect 
algebras, then a homomorphism $h: E \rightarrow F$ is said to be a $\sigma$-homomorphism if $h\left(a_{n}\right) \nearrow h(a)$ whenever $a_{n} \nearrow a$ in $E$.

REMARK 3.3. As a corollary of Theorem 3.2, in view of the basic representation of effect algebras with (RDP), Theorem 2.1 and Remark 2.2, we have that if $E$ is a monotone $\sigma$-complete effect algebra with (RDP), then $E=\Gamma(G, u) \cong \Gamma(A, 1) \cong$ $\left\{f \in \operatorname{Aff}(\mathscr{S}(E)): 0 \leq f \leq 1, f(s) \in s(E)\right.$ for all discrete $\left.s \in \operatorname{Ext}_{\mathscr{S}}(E)\right\}$.

\section{Loomis-Sikorski theorem, effect-tribes, and monotone $\sigma$-complete effect algebras}

In the present section, we show that under some natural conditions, a monotone $\sigma$-complete effect algebra with (RDP) is a $\sigma$-homomorphic image of an effect-tribe (as monotone $\sigma$-complete effect algebras), which is a monotone $\sigma$-complete effect algebra of fuzzy sets. This effect-tribe can be chosen to be a system of affine fuzzy sets defined on a compact, convex, Hausdorff topological space. The conditions will entail that our monotone $\sigma$-complete effect algebras are in fact only all $\sigma$-complete MV-algebras.

An effect-tribe on a set $\Omega \neq \emptyset$ is any system $\mathscr{T} \subseteq[0,1]^{\Omega}$ such that

(i) $1 \in \mathscr{T}$;

(ii) if $f \in \mathscr{T}$, then $1-f \in \mathscr{T}$;

(iii) if $f, g \in \mathscr{T}, f \leq 1-g$, then $f+g \in \mathscr{T}$; and

(iv) for any sequences $\left\{f_{n}\right\}$ of elements of $\mathscr{T}$ such that $f_{n} \nearrow f$ (pointwisely), then $f \in \mathscr{T}$.

It is evident that any effect-tribe is a monotone $\sigma$-complete effect algebra.

It is clear that given a family, $B$, of fuzzy sets on $\Omega$, there is a minimal effect-tribe, $\mathscr{T}_{0}(B)$, on $\Omega$ generated by $B$.

In particular, if $B$ is a system of affine functions on the convex space $\Omega$, so is $\mathscr{T}_{0}(B)$.

We recall that a topological space $X$ is said to be basically disconnected provided the closure of every open $F_{\sigma}$ subset of $X$ is open.

For a bounded function $g: X \rightarrow \mathbb{R}$ on a topological space $X$, we define

$$
\tilde{g}(x)=\inf _{U \in, \mathcal{N}(x)} \sup \{g(y): y \in U\},
$$

where $\mathscr{N}(x)$ is the system of open neighbourhoods for $x \in X$. Then

(i) $g(x) \leq \tilde{g}(x)$ for any $x \in X$.

(ii) $\tilde{g}(x)=g(x)$ if $g$ is continuous in $x$. 
If $D(g)$ is the set of discontinuity points for $g$, then

$$
\{x \in X: g(x) \neq \tilde{g}(x)\} \subseteq D(g)=\bigcup_{n}\left\{g^{-1}\left(R_{n}\right)-\operatorname{Int}\left(g^{-1}\left(R_{n}\right)\right)\right\},
$$

where $\left\{R_{n}\right\}$ is an open basis in $\mathbb{R}$.

Let $f$ be a real-valued function on $\Omega \neq \emptyset$ and $X$ a nonempty subset of $\Omega$. We define $N_{X}(f):=\{x \in X:|f(x)|>0\}$.

Now we are able to present the main result-the Loomis-Sikorski theorem for special monotone $\sigma$-complete effect algebras. As we will see in Lemma 4.2, our $E$ is in fact a $\sigma$-complete $\mathrm{MV}$-algebra and vice versa.

THEOREM 4.1 (Loomis-Sikorski). Let $E$ be a monotone $\sigma$-complete effect algebra with (RDP) such that there is a compact basically disconnected subset $X$ of $\operatorname{Ext}_{\mathscr{S}}(E)$ containing all discrete extremal states of $E$ which is determining. Then there are a convex space $\Omega$, an effect-tribe $\mathscr{T}$ of affine fuzzy sets on $\Omega$ and $a \sigma$-homomorphism $h$ from $\mathscr{T}$ onto $E$.

ProOF. Due to Theorem 2.1, $E=\Gamma(G, u)$ for some interpolation group $(G, u)$ with strong unit. As it was said above, $\mathscr{S}(E)$ is affinely homeomorphic with $\mathscr{S}(G, u)$, and $\operatorname{Ext}_{\mathscr{S}}(E)$ with $\operatorname{Ext}_{\mathscr{S}}(G, u)$. Hypotheses imply by (2) of Theorem 2.3 that $(G, u)$ is monotone $\sigma$-complete, therefore, by Theorem $3.2, E$ is isomorphic with $\Gamma(A, 1)$ (as effect algebras), where $(A, 1)$ is the set of all continuous affine functions $f$ on $\mathscr{S}(G, u)$ such that $f(s) \in s(G)$ for any discrete state $s$ on $(G, u)$. Consequently, by Proposition 3.1, every element $a \in E$ can be identified as an affine continuous function $\hat{a}(\hat{a}(s)=s(a), s \in \mathscr{S}(E))$ and taking values in the real interval $[0,1]$, see Remark 3.3.

Let $\mathscr{T}$ be the effect-tribe of fuzzy sets on $\Omega:=\mathscr{S}(E)$ generated by the set of continuous affine functions $\hat{E}=\{\hat{a}: a \in E\} . \Omega$ is a non-void compact Hausdorff space.

Consider by $\mathscr{T}^{\prime}$ the class of all functions $f \in \mathscr{T}$ with the property that for some $b \in E, N_{X}(f-\hat{b})$ is a meager subset of the basically disconnected compact Hausdorff space $X$.

(i) If $b_{1}$ and $b_{2}$ are two elements of $E$ such that $N_{X}\left(f-\hat{b}_{i}\right)$ is a meager subset for $i=1,2$, then $N_{X}\left(\hat{b}_{1}-\hat{b}_{2}\right) \subseteq N_{X}\left(\hat{b}_{1}-f\right) \cup N_{X}\left(f-\hat{b}_{2}\right)$ is a meager subset of $X$. Due to the Baire theorem saying that any nonempty open set of a compact Hausdorff space cannot be a meager set, we conclude that $\hat{b}_{1}(s)=\hat{b}_{2}(s)$, for any $s \in X$, that is, $b_{1}=b_{2}$ because $X$ is determining.

(ii) It is clear that $\mathscr{T}^{\prime}$ is closed under the formation of complement $f \mapsto 1-f$, and it contains $\{\hat{a}: a \in E\}$. 
(iii) Let $\left\{f_{n}\right\}_{n}$ be a sequence of non-decreasing elements from $\mathscr{T}^{\prime}$. Choose $b_{n} \in E$ such that $N_{X}\left(f_{n}-\hat{b}_{n}\right)$ is a meager subset of $X$. We assert that $b_{1} \leq b_{2} \leq \cdots$, and the sequence $\left\{b_{n}\right\}$ is unique.

Indeed, we set $h_{1}=\hat{b}_{1}$ and for any integer $n \geq 2$, by [7, Corollary 11.16], there is an affine continuous function $h_{n}$ such that $h_{n} \geq h_{n-1}, \hat{b}_{n}$ and $h_{n}(s)=$ $\max \left\{h_{n-1}(s), \hat{b}_{n}(s)\right\}, s \in X$. From the construction we see that every $h_{n} \in A$, and $N_{X}\left(f_{n}-h_{n}\right)$ is meager. Therefore, by (i) we can assume that $h_{n}=\hat{b}_{n}$ which implies $b_{1} \leq b_{2} \leq \cdots$.

(iv) If $f, g \in \mathscr{T}^{\prime}, f \leq 1-g$, and $N_{X}(f-\hat{a}), N_{X}(g-\hat{b})$ are meager subsets, then by (iii) $a \leq b^{\prime}$, so that $a+b \in E$. Hence, $N_{X}(f+g-(\widehat{a+b}))$ is meager, that is, $f+g \in \mathscr{T}^{\prime}$, and $\mathscr{T}^{\prime}$ is an effect algebra.

(v) To show that $\mathscr{T}^{\prime}$ is an effect-tribe is necessary to verify that $\mathscr{T}^{\prime}$ is closed under limits of non-decreasing sequences from $\mathscr{T}^{\prime}$. Let $\left\{f_{n}\right\}_{n}$ be a sequence of nondecreasing elements from $\mathscr{T}^{\prime}$. For any $f_{n}$, choose by (iii) a unique $b_{n} \in E$ such that $N_{X}\left(f_{n}-\hat{b}_{n}\right)$ is a meager subset of $X$.

Denote by $f=\lim _{n} f_{n}, b=\bigvee_{n=1}^{\infty} b_{n}, b_{0}=\lim _{n} \hat{b}_{n}$. Then $f, b_{0} \in \mathscr{T}$ and $b \in E$. We have

$$
N_{X}(f-\hat{b}) \subseteq N_{X}\left(f-b_{0}\right) \cup N_{X}\left(\hat{b}-b_{0}\right)
$$

and

$$
N_{X}\left(f-b_{0}\right)=\left\{s \in X: f(s)<b_{0}(s)\right\} \cup\left\{s \in X: b_{0}(s)<f(s)\right\} .
$$

If $s \in\left\{s: f(s)<b_{0}(s)\right\}$, then there is an integer $n \geq 1$ such that $f(s)<\hat{b}_{n}(s) \leq$ $b_{0}(s)$. Hence $f_{n}(s) \leq f(s)<\hat{b}_{n}(s) \leq b_{0}(s)$ so that $s \in\left\{s: f_{n}(s)<\hat{b}_{n}(s)\right\}$.

Similarly we can prove that if $s \in\left\{s: b_{0}(s)<f(s)\right\}$, then there is an integer $n \geq 1$ such that $s \in\left\{s: \hat{b}_{n}(s)<f_{n}(s)\right\}$.

The last two cases imply $N_{X}\left(f-b_{0}\right) \subseteq \bigcup_{n=1}^{\infty} N_{X}\left(\hat{b}_{n}-f_{n}\right)$ which is a meager subset of $X$.

Apply now (4.1) to the function $b_{0}$ to obtain $\tilde{b}_{0}$, that is,

$$
\tilde{b}_{0}(s):=\inf _{U \in \mathcal{N}(s)} \sup \left\{b_{0}(y): y \in U\right\}, \quad s \in X .
$$

Since $X$ is basically disconnected, compact, Hausdorff, and

$$
b_{0}^{-1}(\alpha, \infty)=\bigcup_{n} \hat{b}_{n}^{-1}(\alpha, \infty)
$$

for any $\alpha \in \mathbb{R}, b_{0}^{-1}(\alpha, \infty)$ is an open $F_{\sigma}$ set in $X$, see [7, Lemma 9.1], $\tilde{b}_{0}$ is continuous on $X$. Since $b_{0}$ is the point limit of a sequence of continuous functions, by $[9$, pages 86 , 405-6], $D\left(b_{0}\right) \supseteq N_{X}\left(\tilde{b}_{0}-b_{0}\right)$ is a meager subset in $X, b_{0}(s) \leq \tilde{b}_{0}(s) \leq \hat{b}(s), s \in X$, and $N_{X}\left(\hat{b}-b_{0}\right) \subseteq N_{X}\left(\hat{b}-\tilde{b}_{0}\right) \cup D\left(b_{0}\right)$. 
By the Tietze theorem for affine continuous functions, [7, Theorem 11.14], there is an affine continuous function $b_{0} \approx$ on $\Omega$ such that $b_{0} \approx(s) \leq \tilde{b}_{0}(s)$ for all $s \in X$ and

$$
b_{0}(s) \leq b_{0}^{\approx}(s) \leq \hat{b}(s), s \in \Omega .
$$

Finally, we show $b_{0}^{\approx}=\hat{b}$. Define $\operatorname{Aff}(\mathscr{S}(G, u))$ as the set of all continuous functions on $\mathscr{S}(G, u)(\cong \mathscr{S}(E))$. We have $\phi\left(b_{n}\right) \leq b_{0} \approx$ for any $n \geq 1$, where $\phi$ is the natural embedding of $(G, u)$ into $\operatorname{Aff}(\mathscr{S}(G, u))$. Since $b_{0} \approx$ is continuous and affine, by [7, Lemma 16.17], there is an element $x \in G$ such that $\phi(x) \leq b_{0} \approx$ and $b_{n} \leq x$ for each $n$. Since $b=\bigvee_{n=1}^{\infty} b_{n}$, we conclude that $\hat{b}=\phi(b) \leq b \approx$.

Consequently, we have proved that $\mathscr{T}^{\prime}$ is an effect-tribe, and whence, $\mathscr{T}^{\prime}=\mathscr{T}$.

Due to the definition of $\mathscr{T}^{\prime}$, for any $f \in \mathscr{T}$ there is a unique element $h(f):=b \in E$ such that $N_{X}(f-\hat{b})$ is meager in $X$, which proves that $h: \mathscr{T} \rightarrow E$ is a surjective $\sigma$-homomorphism in question.

Since $\hat{E}$ consists of affine functions, $\mathscr{T}$ consists also from affine fuzzy sets.

QUESTION. Does $\mathscr{T}$ satisfy (RDP)?

Every $\sigma$-complete MV-algebra $M$ satisfies the conditions of Theorem 4.1, when $X=\operatorname{Ext}_{\mathscr{S}}(M)$. However, our effect-tribe of affine functions is not necessarily an MV-algebra. For such a representation see the next section where instead of the effect-tribe we use the tribe of fuzzy sets. In the following lemma we show that every monotone $\sigma$-complete effect algebra from Theorem 4.1 is in fact a $\sigma$-complete $\mathrm{MV}$-algebra and vice versa.

LEMMA 4.2. Let $E$ be a monotone $\sigma$-complete effect algebra with (RDP). The following statements are equivalent:

(i) E is a lattice.

(ii) There is a nonempty determining set $X \subseteq \operatorname{Ext}_{\mathscr{S}}(E)$ which is compact and basically disconnected.

(iii) $\operatorname{Ext}_{\mathscr{S}}(E)$ is compact.

Proof. Assume $E=\Gamma(G, u)$, where $(G, u)$ is an interpolation group with strong unit. By [7, Corollary 16.28], (i) and (iii) are equivalent.

(i) $\Rightarrow$ (ii). If $E$ is a lattice, then $E$ is in fact a $\sigma$-complete $M V$-algebra. Therefore the set $X=\operatorname{Ext}_{\mathscr{S}}(E)$ is compact, basically disconnected ([7, Corollary 9.10]). Since $E$ is a $\sigma$-complete lattice, $X$ is determining by the Krein-Mil'man theorem.

(ii) $\Rightarrow$ (i). We use the notation from the proof of Lemma 2.5. Let $H=\operatorname{Ker}(\hat{X})$. Then $H$ is an o-ideal of $G$ and by [7, Theorem 16.30], $G / H$ is Dedekind $\sigma$-complete. Since $X$ is determining, $H=\operatorname{Ker}(\hat{X})=\phi_{G}(\operatorname{Ker}(X))=\{0\}$, that is, $G=G / H$ is a lattice, which implies that $E$ is a lattice. 
We recall that $\mathscr{E}(H)$ is a monotone $\sigma$-complete effect algebra which is neither a lattice nor with (RDP). Nevertheless, $\mathscr{E}(H)=\Gamma(\mathscr{B}(H), I)$, where $\mathscr{B}(H)$ is the system of all Hermitian operators on $H$, and it can be isomorphically represented as the effect-tribe $\mathscr{T}(H)=\left\{f_{A}: A \in \mathscr{E}(H)\right\}$, where $f_{A}$ is the fuzzy set on the system of all unit vectors $x \in H$ defined by $f_{A}(x)=(A x, x),\|x\|=1$.

Such an isomorphic effect-tribe $\mathscr{T}$ exists for every monotone $\sigma$-complete effect algebra admitting a (convex, if $\mathscr{T}$ has to consists of affine fuzzy sets) order-preserving system of $\sigma$-complete states. On the other hand it is well-known that there exists even a Boolean $\sigma$-complete algebra admitting no $\sigma$-additive state.

\section{Loomis-Sikorski theorem, affine functions, and $\sigma$-complete MV-algebras}

The Loomis-Sikorski theorem for $\sigma$-complete MV-algebras was proved in [11] and [3] showing that every $\sigma$-complete $\mathrm{MV}$-algebra is an MV- $\sigma$-homomorphic image (as $\sigma$-complete $\mathrm{MV}$-algebras) of a tribe, a special system of fuzzy sets forming a $\sigma$-complete MV-algebra defined on a compact, basically disconnected, Hausdorff topological space.

In the former section we have in fact proved any $\sigma$-complete $\mathrm{MV}$-algebra is a $\sigma$-homomorphic image (as $\sigma$-complete effect algebras) of an effect-tribe which is a monotone $\sigma$-complete effect algebra of affine fuzzy sets defined on a compact, convex, Hausdorff topological space.

Using ideas of the previous section, we now show that the tribe can be chosen to be generated by continuous affine fuzzy sets defined on a compact, convex, Hausdorff topological space.

We recall that a tribe on $\Omega \neq \emptyset$ is a collection $\mathscr{T}$ of fuzzy sets from $[0,1]^{\Omega}$ such that

(i) $1 \in \mathscr{T}$;

(ii) if $f \in \mathscr{T}$, then $1-f \in \mathscr{T}$; and

(iii) if $\left\{f_{n}\right\}_{n}$ is sequence from $\mathscr{T}$, then $\min \left\{\sum_{n=1}^{\infty} f_{n}, 1\right\} \in \mathscr{T}$.

It is possible to show, see for example [4, Proposition 7.1.6], that if $\mathscr{T}$ is a tribe, then (i) $a \vee b=\max \{a, b\} \in \mathscr{T}, a \wedge b=\min \{a, b\} \in \mathscr{T}$, (ii) $b-a \in \mathscr{T}$ if $a \leq b$, that is, if $a(\omega) \leq b(\omega)$ for all $\omega \in \Omega$, (iii) if $a_{n} \in \mathscr{T}$, and $a_{n} \nearrow a$ (pointwisely), then $a=\lim _{n} a_{n} \in \mathscr{T}$, and (iv) $\mathscr{T}$ is a $\sigma$-complete MV-algebra closed under point suprema of sequences from it. We recall that every tribe is an effect-tribe, the converse is not true, in general. We also recall that the $\mathrm{MV}$-algebra $\mathscr{T}=\left(\mathscr{T}, \oplus,{ }^{*}, 0,1\right)$ is defined via $(f \oplus g)(\omega)=\min \{f(\omega)+g(\omega), 1\}, \omega \in \Omega, f^{*}=1-f$.

We are now ready to present a new version of the Loomis-Sikorski theorem for $\sigma$-complete MV-algebra. 
THEOREM 5.1. For every $\sigma$-complete $M V$-algebra $M$, there exist a convex set $\Omega \neq$ $\emptyset$, a tribe $\mathscr{T}$ generated by affine fuzzy sets on $\Omega$, and an $M V$ - $\sigma$-homomorphism $h$ from $\mathscr{T}$ onto $M$.

ProOF. Let $\Omega=\mathscr{S}(M)$. Every $\sigma$-complete $\mathrm{MV}$-algebra is a monotone $\sigma$-complete effect algebra with (RDP). Define $X=\operatorname{Ext}_{\mathscr{S}}(M)$. Then $X$ is a nonempty determining system of extremal states on $M$ which is a compact, basically disconnected, Hausdorff space (see for example Lemma 4.2). Therefore, the conditions of Theorem 4.1 are satisfied.

We repeat the proof of Theorem 4.1 changing the effect-tribe to the tribe and $E$ to $M$. For $f \in \mathscr{T}$ and $a \in M$, we write $f \sim a$ if $N_{X}(f-\hat{a})$ is a meager subset of $X$. Then (i) if $f \sim a$ and $g \sim b$, then $f \oplus g \sim a \oplus b$, (ii) if $f \sim a$, then $1-f \sim a^{*}$, (iii) $\mathscr{T}=\mathscr{T}^{\prime}$, (iv) the mapping $h: \mathscr{T} \rightarrow M$, defined by $h(f)=a$ if $f \sim \hat{a}$, is a surjective MV- $\sigma$-homomorphism.

From the construction we see that $\hat{M}$ is the system of affine continuous functions on $\Omega$ generating the tribe $\mathscr{T}$.

\section{Conclusion}

In the paper, we have proved that every $\sigma$-complete MV-algebra can be represented via the Loomis-Sikorski way as a $\sigma$-homomorphic image of (i) an effect-tribe (as monotone $\sigma$-complete effect algebras) of affine fuzzy sets on a convex, compact, Hausdorff space, Theorem 4.1, or of (ii) a tribe (as $\sigma$-complete MV-algebras) of fuzzy sets generated by a system of affine continuous fuzzy sets on a convex, compact, Hausdorff space, Theorem 5.1.

\section{References}

[1] C. C. Chang, 'Algebraic analysis of many valued logics', Trans. Amer. Math. Soc. 88 (1958), $467-490$.

[2] A. Dvurečenskij, 'Perfect effect algebras are categorically equivalent with Abelian interpolation po-groups', preprint.

[3] _ 'Loomis-Sikorski theorem for $\sigma$-complete MV-algebras and $\ell$-groups', J. Austral. Math. Soc. Ser. A 68 (2000), 261-277.

[4] A. Dvurečenskij and S. Pulmannová, New trends in quantum structures (Kluwer Acad. Publ., Dordrecht, Ister Science, Bratislava, 2000).

[5] A. Dvurečenskij and T. Vetterlein, 'Pseudoeffect algebras. II. Group representation', Int. J. Theor. Phys. 40 (2001), 703-726.

[6] D. J. Foulis and M. K. Bennett, 'Effect algebras and unsharp quantum logics', Found. Phys. 24 (1994), 1325-1346. 
[7] K. R. Goodearl, Partially ordered Abelian groups with interpolation, Math. Surveys Monogr. 20 (Amer. Math. Soc., Providence, RI, 1986).

[8] F. Kôpka and F. Chovanec, 'D-posets', Math. Slovaca 44 (1994), 21-34.

[9] K. Kuratowski, Topology I, (in Russian) (Mir, Moskva, 1966).

[10] D. Mundici, 'Interpretation of AF $C^{*}$-algebras in Lukasiewicz sentential calculus', J. Funct. Anal. $65(1986), 15-63$.

[11] _ 'Tensor products and the Loomis-Sikorski theorem for MV-algebras', Adv. Appl. Math. 22 (1999), 227-248.

[12] K. Ravindran, On a structure theory of effect algebras (Ph.D. Thesis, Kansas State Univ., Manhattan, Kansas, 1996).

Mathematical Institute

Slovak Academy of Sciences

Štefánikova 49

SK-814 73 Bratislava

Slovakia

e-mail: dvurecen@mat.savba.sk 